\title{
A New Experimental Limit on the Electric Dipole Moment of the Electron
}

\author{
C. Carlberg and $\mathrm{H}$. Gould \\ Materials and Chemical Sciences Division \\ Lawtence Berkeley Laboratoiy \\ University of Califormia \\ Berkeley, California 94720 \\ K. Abdullah, E.D. Commins, and S.B. Ross \\ Department of Physics \\ University of Califarnia \\ and \\ Materials and Chemical Sciences Division \\ Lawrence Berkeley Laboratory \\ University of California \\ Berkeley, Califomia 94720
}

\section{December 1990}

\section{DISCLAIMER}

\begin{abstract}
This report was prepared as an acoount of work sponsored by an agency of the Uniled Sitates Government. Neitber the United States Government nor any agency thercof, nor any of their employecs, makes any warranty, express or implied, or assumes any legal liabitity or responsibility for the accuracy, completeness, or usefulness of any information, apparatus, product, or process disclased, or represents that its use would not infringe privately owned rights. Refer. ence berein to any specific commercial product, process, or service by trade name, trademark, manufacturer, or otherwise does not necessarily constitute or imply its endersement, recommendatien, or favoring by the United States Government or any agency thereol. The views and opinions of authors expressed herein do not necesearily state or reflect thase of the United States Gavernment or any agency thereof.
\end{abstract}

This work wis supported by the Direclor, Office of Energy Research, Office of Basic Energy Scionces, Chemical Sciences Division; and in part by the Office of High Energy and Nuclear Physics, Division of High Energy Physics and Division of Nuclear Physics, of the U.S. Department of Energy under Contract No. DE-AC03. $76 \mathrm{SF} 00098$. 


\title{
A NEW EXPERIMENTAL UMIT ON THE ELECTPAC DIPOLE MOMENT OF THE ELECTRON C.Carlberg ${ }^{1, a}, K_{\text {. Abdullah }}{ }^{1,2, b}$, E. D.Commins ${ }^{1,2}$, H. Gould ${ }^{1}$, and S.B. Ross ${ }^{1,2}$ $1_{\text {Materials and Chemical Scientes Division }}$ \\ Lawrence Berkeley Laboratory \\ University of California \\ Berkeley, California 94720 \\ and \\ 2Department of Physics \\ University of California \\ Berkeley, California 94720
}

\begin{abstract}
We describe a search for the electric dipole moment $d_{e}$ of the electron, carried out with ${ }^{205} \mathrm{Tl}$ atoms in the ground state. The experiment makes use of the separatedoscillating-field magnetic-resonance method, laser state selection, fluorescence detection, and two counter-propagating atomic beams. Very careful attention is paid to systematic effects. The result for the atomic electric dipole moment is $d_{a}=(1.6 \pm 5.0) \times 10^{-24} e \mathrm{~cm}$. If we assume the theoretical ratio $d_{a} / d_{e}=-600$, this yields $d_{e}=(-2.7 \pm 8.3) \times 10^{-27}$ e cm.
\end{abstract}

\section{INTRODUCTION}

We have carried out a new search for the electric dipole moment (EDM) of the electron, a moment that can exist only by virtue of an interaction that violates parity $(P)$ and time reversal invariance $(T)$. The present investigation is thus of interest in connection with $\mathrm{CP}$ violation. Indeed, a number of theoretical models of $\mathrm{CP}$ violation have recently appeared ${ }^{1-s}$, that predict the existence of an electron EDM in the range $10^{-26}$ $-10^{-27} \mathrm{e} \mathrm{cm}$, possibly large enough to be detected in a practical experiment.

The general idea in any EDM search is 10 detect a dipole moment $d$ by measuring its energy $W$ in an electric field $E$ :

$$
W=\cdot d \cdot E
$$

but obviously this cannot be done with free electrons, which are charged. One thinks instead about an unpaired valence electron in a neutral atom. The question arises: can the atom as a whole possess an EDM $d_{a}$ proportional to the electron EDM $d_{e}$ ? Schiff's theorem 5 , based on non-relativistic quantum mechanics, states that, to first order in $d_{a}, d_{a}$ is zero. However, relativistic effects are important in atoms with large atomic number, and the ratio $R=d_{a} / d_{\theta}$, roughily proportional to $z^{3} \alpha^{2}$, can in fact be much larger than unity 6 . Thus, we actually search for $d_{a}$ in the $6^{2} P_{1 / 2}$ ground state of 
thallium $(Z=81)$, where $R$ is estimated 7.10 to be approximately -600 , and interpret the result in terms of $d_{e}$.

\section{EXPERIMENTAL METHOD}

\subsection{Ihallium Atomic Beams}

Our experiment utilizes two vertical atomic beams of thallium, one travelling upward and the other downward (see Fig. 1). This specific feature of a pair of beams with opposite velocities is essential: it permits virtually complete elimination of an important systematic effect: that associated with the interaction of the atomic magnetic moriient with the motional magnetic field Exv/c; (see Sec. 4.2). The beams are generated in conventional stainless steel ovens that operate at about $1050^{\circ} \mathrm{K}$. The beam velocity distributions closely approximate $v^{3}$ Maxwellians, but the beams effuse from rather deep rectangular source slits. Thus their effective temperatures are about $1500^{\circ} \mathrm{K}$, corresponding to most-probable velocities of about $4.3 \times 10^{4} \mathrm{~cm} / \mathrm{s}$. The beam trajectories are defined by various collimating slits to be described below, anc are vertical rather thari horizontal to avoid deflection from failing in the earth's gravitational fieid. The measurements are performed on one atomic beam at a time with two computer controlled beam stops switching between them.

\subsection{State Selection}

Let Ls follow the progress of the upward-going beam in Fig. 1. After emerging from the oven, and moving in the positive $x$ direction, this beam intersects a $\mathrm{cw}$ laser beam directed along $y$ and with linear polarization along $z$ in the state selector region. Note that the entire experiment from here on takes place in a nominally uniform magnetic field $B_{2}$. typically .26 Gauss or less, in the 2 direction. The laser is turied to the allowed E1 transition $6^{2} \mathrm{P}_{1 / 2}(\mathrm{~F}=1) \rightarrow 7^{2} \mathrm{~S}_{1 / 2}(\mathrm{~F}=1)$ at $378 \mathrm{~nm}$ (See Fig. 2). Ground state atoms with $m_{F}= \pm 1$ can make the optical transition, but the matrix element for $F=1, m_{F}=0 \rightarrow F=1, m_{F}=0$ is zero. Atoms excited to the $7 S$ state decay rapidly by spontaneous emission to $6 \mathrm{P}_{1 / 2}$ or to $6 \mathrm{~F}_{3 / 2}$ (with fluorescence at $535 \mathrm{~nm}$ ): the branching ratio being about $50 \%$. Furthermore, the $6 \mathrm{P}_{3 / 2}$ state has a lifetime much longer than the beam flight time through the apparatus. Consequently optical pumping depopulates the ground state components $F=1, m_{F}= \pm 1$, and increases the population of $F=1, m_{F}=0$ (by a factor of 1.4). As $F=1$ atoms leave the state selector they are thus in the pure state $m_{F}=0$, represented by:

$$
\Psi=\left(\begin{array}{l}
0 \\
1 \\
0
\end{array}\right)
$$




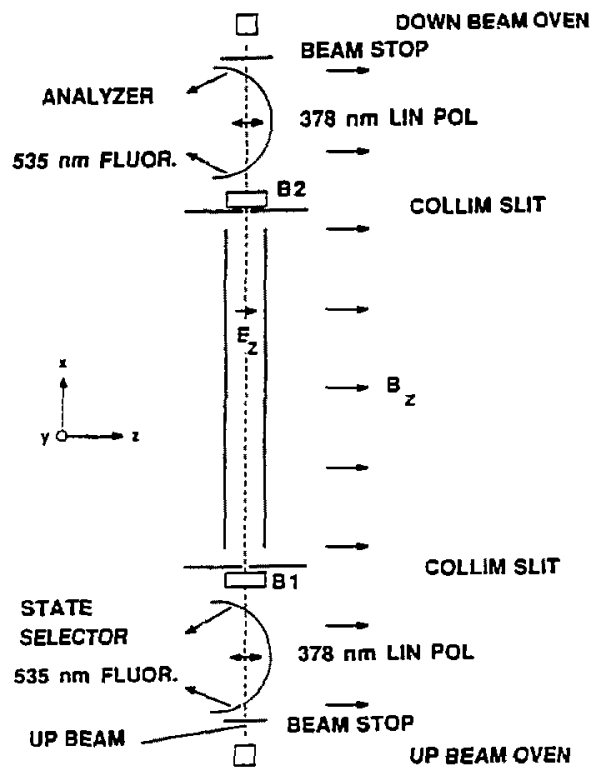

Fig. 1 Schematic diagram of experimental apparatus.

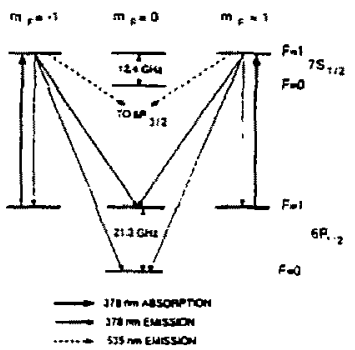

Fig .2 Energy level diagram showing optical state selection scheme. 
Further along the atomic beam trajectory is a second optical pumping region identical to the first, called the analyzer. Here also, the atomic beam intersects a beam from the same laser directed along $y$ with linear polarization along $z_{\text {, and tuned to the }}$ same transition $6 P_{1 / 2}, F=1 \rightarrow 7 S_{1 / 2}, F=1$. The analyzer fluorescent signal at $535 \mathrm{~nm}$ is proportional to the sum of the populations of $F=1, m_{F}= \pm 1$ atoms that enter this region.

The laser beam-atomic beam intersection in the state selector or analyzer occurs at the focus of a large reflecting ellipsoid with its axis of revolution along z. Its purpose is to collect $535 \mathrm{~nm}$ fluorescence and focus it through a light pipe/opticaf filter sysiem inio a photomultiplier tube.

Fig. 3 shows the state selector fluorescent signal versus laser frequency in the neighborhood of the $F=1 \rightarrow F=1$ resonance, and shows that the two naturally occurring thallium isotopes $A=203,205$ (with abundances 30\%,70\%) are resolved by the isotope shift. All our EDM measurements are carried out with the more abundant isotope $A=205$.

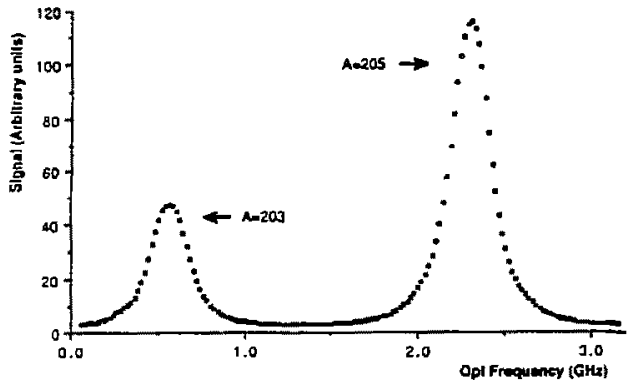

Fig. 3 Fluorescent signal at $535 \mathrm{~nm}$ in state selector versus optical frequency, for the $F=1 \rightarrow F=1378 \mathrm{~nm}$ transition in isotopes $A=203, A=205$.

Fig. 4 is a plot of stale selector and analyzer fluorescent signals (with different gains) on the $A=2051 \rightarrow 1$ line and with no reorientation in the region between state selector and analyzer. The shape of the analyzer signal, in particular the flat botiom of the curve at resonance, is easy to understand: in the polarizer, all $m_{F}= \pm 1$ ground state atoms are removed, and thus there are no $m_{F}= \pm 1$ atoms entering the analyzer to generate a fluorescent signal at resonance. Such a signal will only occur if the $F=1$ state is reorienfed (by if and electric field) in the intervening space. Note also the oplical linewidth: it is much broader than the natural width of $21 \mathrm{MHz}$, because of optical power 
broadening (saturation).

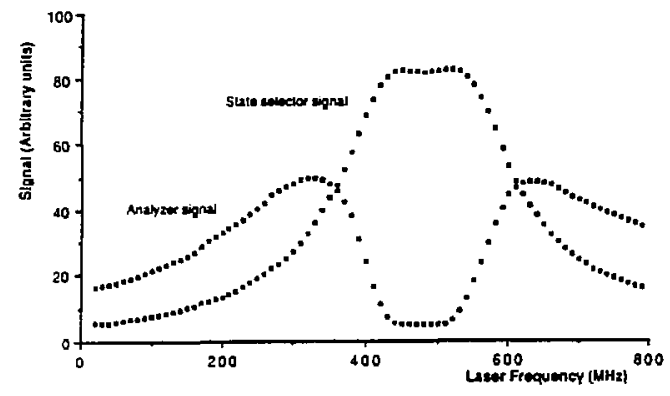

Fig. 4 Fluorescent signais at $535 \mathrm{~nm}$ in state solector and analyzer (with different gains) versus optical frequency, for the $F=1 \rightarrow F=1378 \mathrm{~nm}$ transition, $A=205$.

The state selector ior the up-going beam serves as the analyzer for the down-going beam, and vice versa.

The $378 \mathrm{~nm}$ light is supplied by a Coherent CR 699-21 dye laser with intra-cavity doubling ( $\left(\mathrm{HiO}_{3} \mathrm{crystal}\right)$, pumped by a Coherent $100-\mathrm{K} 3 \mathrm{Krypton}$ laser. Typically we obtain about 2-3 $\mathrm{mW}$ of single mode $378 \mathrm{~nm}$ optical power.

\subsection{Badio frequency transitions.}

Let us continue to follow the upgoing beam after it leaves the state selector (Fig.1). Before reaching the analyzer it passes through two separated of magnetic tields $B_{1}, B_{2}$ (Ramsey Method) ${ }^{11}$ and, in the space between them, an electric field $E$ (typically 100 $k V / c m$ ) in the $z$ direction and a pair of collimating slits $C_{1}, C_{2}$. Each if region has length $a=5 \mathrm{~cm}$, and $B_{1}, B_{2}$ oscillate coherently in the $x$ direction with frequency $i$ near the resonant irequency $\omega_{0}$ for the transitions $F=1, m_{F}=0 \cdot m_{F}= \pm 1$ in constant magnetic field $B_{z}$. $B_{2}$ leads $B_{1}$ by phase $\alpha$, which is $\pm \pi / 4$ or $\pm 3 \pi / 4$. The collimating slit dimensions are $0.5 \mathrm{~cm} \times 0.05 \mathrm{~cm}$ in the $y$ and $z$ dimensions respectively.

In $B_{1}$ the pure $F=1, m_{F}=0$ state is converted to a coherent superposition of $m_{F}$ states. For an atom with velocity $v$, and at resonance, one finds that in the rotating frame the wave lunction immediately after the first if region has changed from (2) 10: 
where

$$
\psi=\left(\begin{array}{c}
\frac{-i \sigma}{\sqrt{2}} \\
\eta \\
\frac{-i \sigma}{\sqrt{2}}
\end{array}\right)
$$

and $\gamma=\frac{\mu_{0} \mid g F}{\sqrt{\pi}}$. Wave-function (3) evolves further in the electric field region. After the atom passes through the E field, (3) becomes:

$$
\Psi=\left(\begin{array}{c}
\frac{-i \sigma}{\sqrt{2}} e^{-i \varepsilon} \\
\eta e^{i \phi} \\
\frac{-i \sigma}{\sqrt{2}} e^{i \varepsilon}
\end{array}\right)
$$

Her $\phi=\frac{k E^{2}}{v}$ arises from the hypertine-induced quadratic Stark shift (about $400 \mathrm{~Hz}$ at $100 \mathrm{kv} / \mathrm{cm}$ ) of $m_{F}=0$ relative to $m_{F}= \pm 1 ; k$ is a constant that has been measured and calculated. (Actually there is also a very small hyperfine/Zeeman-induced quadratic Stark shif between $m_{F}=1$ and $m_{F}=-1$ levels that is proportional to $B_{z}$; however this effect is of negligible importance). The quantity $\varepsilon$ is given by:

$$
\varepsilon=\varepsilon_{0}+\varepsilon^{\prime}
$$

where

$$
\varepsilon_{0}=-\frac{d_{A} E}{\hbar} \frac{L}{v}=-\frac{d_{s} R E}{\hbar v}
$$

arises from the EDM, and where $\epsilon^{\prime}$, also odd in $E$, arises from systematic effects to be described below.

The wave function is further modified in the second $i f$ region, and it can be shown that neat resonance and for equa! $B_{1}, B_{2}$ magnitudes the signal in the analyzer-detector is proportional to the following expression:

$$
S=\left\langle A_{1}-A_{2} \cos ^{2}(\Delta \omega T+\alpha \mp \varepsilon)+A_{3} \cos (\Delta \omega T+\alpha \dddot{F})\right\rangle
$$

where $A_{1}=1-\eta^{4}, A_{2}=\sigma^{4}, A_{3}=2 \eta^{2} \sigma^{2} \cos \phi_{1} \Delta \omega=a^{2}-\omega_{0}, T=L^{\prime} / v$ where $L^{\prime}=120 \mathrm{~cm}$ is the distance between $r$ regions, the $\mp$ sign is employed for $B_{2}>0(<0)$ and $<\ldots>$ indicates an average over the velocity distribution. A formula sirnilar to (7) applies for the down beam, except that since the roles of the two if regions are reversed, a must be replaced by $\alpha^{\prime}=-\alpha$. Also, some contributions to $\varepsilon^{\prime}$ have opposite signs for the up and down bearns. The signature of an EDM is the appearance in $S$ of an asymmetry proportional to 
the P,T-odd rotational invariant E*B.

Fig. 5 shows the fiuorescent signal in the analyzer plotted as a function of applied if frequency in the neighborhood of resonance, for $\alpha= \pm 3 \pi / 4$. These observed "Ramsey interference" curves are in excellent agreement with calculations based on the foregoing discussion. Optical resonance curves such as Fig. 4, and if resonance curves such as Fig. 5 are frequently scanned in the course of the experiment.



Fig. 5 Fluorescent signal at $535 \mathrm{~nm}$ in analyzer versus radio frequency in the vicinity of double loop resonance. Phases $\alpha= \pm 3 \pi / 4$ are shown.

\subsection{Electric Fields}

The electric lield is produced with a pair of titanium alloy (90\% Ti, 6\% Al, $4 \% \mathrm{~V}$ ) plates, mounted on a structure with plate spacings maintained by precision-ground fused-silica spacers. The plates are $100 \mathrm{~cm}$ long, $5 \mathrm{~cm}$ wide, and separated by $0.2 \mathrm{~cm}$. Even though they were machined, ground, and assembled with care, there is always a considerable amount of arcing and sparking berween the plates when high voltage is first turned on in vacuum. Presumably this happens because of field emission from plate surface irregularities. The effect is reduced by a lactor of $10^{3}$ or more by runring the plates at high voltage for some hours with argon gas at about $10^{-3}$ torr pressure. Once the field-emission currents have been reduced by argon treatment, the gas is pumped away, and the plates are ready for service until the next time the system is brought up to atmosphere.

\subsection{Magnetic Fields}

As mentioned previcusly, the experiment takes place in a weak, nominally uniform 
magnetic field $B_{z}$ generated by a pair of rectangular coils: In lact the field supplied by these coils is not quite unilorm. To produce a Ramsey resonance that is centered on the broad single-loop resonance, we also employ small "trim" coils 10 generale a correction field $\theta_{z}$ ' at each of 'region. As will be seen below, it is important to cancel the average resicual magnetic field $B_{y}$ at the beam axis. This is accomplished with rectengular coils similar to those for $\mathrm{B}_{z}$. In addition the system is provided with a set of coils 10 generate a magnetic field $\theta_{x}$ in the beam direction, the purpose of which will be described below. Finally there is a set of four wires (called 'inhomogeneity' wires) parallel to the $x$ axis that can be used in any desired combination to generate magnetic fields that have large gradients in the $y$ and $z$ directions at the beam axis. This entire system of coils and wires, and the vacuum system they surround, are enclosed in a set of four nested magnetic shields (Hypernom and Co-Netic). The magnetic shields have been carefully annealed and are deGaussed with auxiliary deGaussing coils after each change of magnetic field. The shields reduce the ambient magnetic field (from the earth and nearby equipment) by a factor of at least $2 \times 10^{4}$ in the $y, z$ direction and $2 \times 10^{3}$ in the $x$ direction.

\section{DATAACQUISITION}

Before we acquire data, the laser frequency is locked to the center of the optical resonance by the computer. The computer than locks the it synthesizer frequency to the center of the Ramsey resonance. The actual EDM asymmetry search then consists of repeated measurements of fluorescent signals in the analyzing region for different experimental conditions (chops).

In order to determine our sensitivity 10 an atomic EDM we must measure what frequency shitt a certain signal difference corresponds to. Therefore we collect half of our data $1 \mathrm{~Hz}$ above the center of double-loop resonance and the other half $1 \mathrm{~Hz}$ below. From this known frequency shift and the measured signal difference it is possible to measure the slope of the resonance and thus calibrate the sensitivity. The latter quantity is continuously constructed from the same data that determines the EDM.

In addition to the calibration stepping of the if frequency there are three more computer controlled chops : 1) The if phase difference $\alpha$ (if phase chop), 2) the electric field polarity reversal ( $E$ chop) and 3 ) the up-down beam reversal (beam chop). The it phase chop minimizes false effects from the quadratic Stark effect and also reduces noise caused by frequency-independent fluctuations such as laser or atomic beam intensity and background variations. Naturally, the purpose of the electric field chop is 10 extract the true EDM, and it is the only chop which averages noise from magnetic fietd fluctuations. As mertioned previously, the beam chop largely eliminates the false EDM asymmelry due to the Exv effect. 
The of phase is chopped after each $0.05 \mathrm{~s}$ of integration among $+45,-45,+135$, and -135 degrees. The electric field is reversed after $0.8 \mathrm{~s}$ of integration by a computer controlled high voltage switch, the it trequency (the calibration chop discussed eartier) is stepped after 3.2. $\mathbf{S}$ of integration, and the direction of the atomic beam is reversed after $6.4 \mathrm{~s}$ of integration. Note that these chops are nested in each other and that, for example, each if phase is repeated four times for one sign of the electric field. After a change of $i$-phase we wait $0.018 \mathrm{~s}$ to insure that atoms which have experienced the previous if-phase have passed through the analyzing region. When the electric field is reversed the experiment pauses for $0.4 \mathrm{~s}$ to let the plates charge up completely before continuing the integration. The same delay $(0.4 \mathrm{~s})$ is also used for the calibration step and when the direction of the atomic beam is reversed. Each photomultiplier signal is amplified with a preamplifier and then integrated for $0.9 \mathrm{~ms}$ by a gated integrator. The output of the integrator is digitized to 12 bits. The computer then stores the result in a temporary siorage array.

Since the signal should be constant within a chop configuration, it is possible to calculate an average signal and its standard deviation from data within a configuration. If the standard deviation is too large, all the data within the actual $+E /-E$ period are rejected and the data-acquisition restarted with the first contiguration in the actual $E$ period. Only after a full E-period is successfully completed are the data stored permanentiy and data-acquisition allowed to start with the next E-period. This rejection occurred only very rarely in actual runs.

Each photomuliplier is alternately exposed to large and small signals because of the beam chop. After a large signal the tube requiras a finite amount of time to recover stability; to prevent this from distoring the small signal systematically we permute the chop start configuration and sequence. After eight full beam periods, corresponding to $104.2 \mathrm{sec}$. of integration and about $4 \mathrm{~min}$. of real time, the acquisition is halled and the current EDM and $\angle B_{y} / B_{z}>$ are displayed. The EDM is proportional to the sum of the up and down beam asymmetries while $\left\langle\theta_{y} / B_{z}\right\rangle$ is proportional to the difference. (The latter arises from the motional magnetic field effect discussed in Sec. 4.2). Then $\theta_{y}$ is corrected (if necessary) to make $\left\langle\mathrm{B}_{\mathrm{y}} / \mathrm{B}_{z}\right\rangle$ as small as possible and the center of the optical resonance is redetermined belore data acquisition is restarted. After a set of sixteen EDM's are measured an average EDM and its standard deviation are calculated. This result is a data point which represents 26 min. of integration and about 1 hour of real time. Our data points are shown in Fig. 6a,b.

When a data point is completed the current to the magnetic field coils is reversed and/or the electric field cables are reversed manually. The purpose of these reversals is to distinguish between odd and even effects under $B$ andior $E$ reversal. These reversals also allow us to isolate false effects caused, for example, by computer control signals and the electric field switch. Our overall data taking efficiency was about $30 \%$ when taking 
into account time needed for waits, rejections, reversals, stabilisations and maintenance.

a)

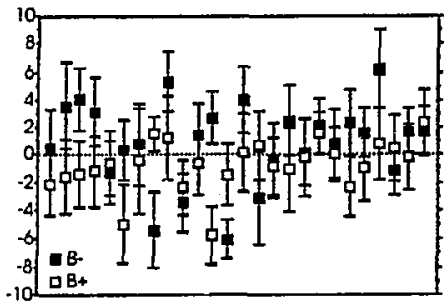

b)

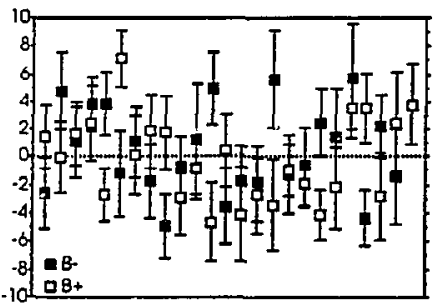

Fig. 6. a) Atomic EDM data of run I. b) Atomic EDM data of tun II. In each case the signs of $B_{\text {. }}$ data points are reversed for comparison with $B_{+}$data points. Vertical scale units:10-23 a cm.

\section{SYSTEMATICS}

Isolation and elimination of systematic errors is of central importance in an experiment such as this one, where an extremely small or zero effect is being measured. In the following section we describe in some detail our principal sources of systematic error, and the auxiliary measurements we have carried out to determine them.

\subsection{The Geometric Phase Effect}

This effect is purely classical, although it requires quantum mechanical interference for observation. It arises when a magnetic field $B_{x}$ exists that assumes different values at the lower and upper ends of the electric field region. It may be understood intuitively by referring to Fig. 7 , which illustrates schematically the experimental set-up we actually use to observe tine phenomenon. 


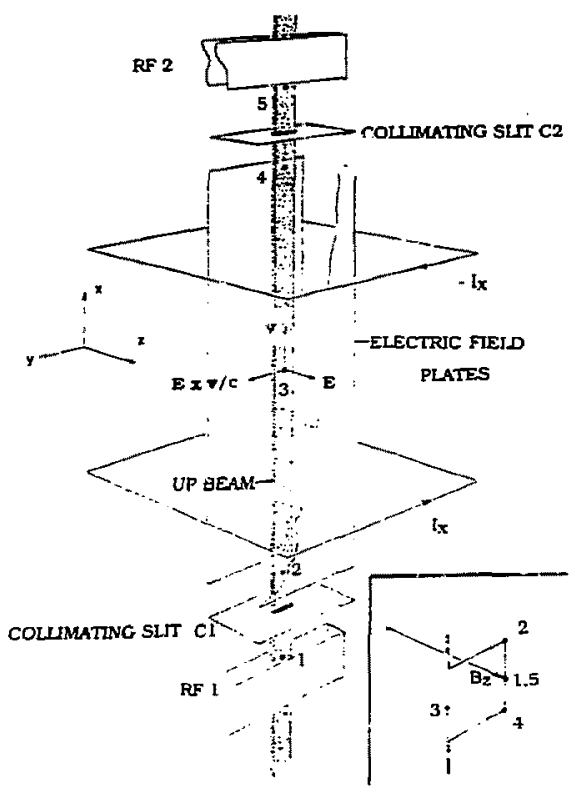

Fig.7 Schematic diagram of experimental setup used to observe the geometric phase effect. Insen shows haw tip of magnetic field vector evolvos with time.

Fig. 7 shows a pair of $x$ coils with equal and opposite currents $I_{x}$ for generating a very inhomogeneous field $B_{x}$ that is positive at the lower end of the electric lieto regiun and negative at the upper end. An atom in the up beam at first experiences a negligible field $\mathrm{B}_{\mathrm{x}}$ (position \# 1). (See also the insert in Fig. 7, which shows the evolulion of the tip of $B$.) As the atom moves closer to the $E$ field region, $8_{x}$ increases. At the entrance to the electric field $\mathrm{B}_{\mathrm{x}}$ is still larger, and the molional field $\mathrm{B}_{\mathrm{y}}{ }^{\prime}=+v$. ese tuins on rapidly but adiabatically(\# 2). By this we mean that the majnetic momenl precesses many times in the length over which $E$ varies between zero to its iull value. As the atom continues to move upward the $B_{x}$ field continues to increase for a time and then decreases to zero at the midpoint of the E field (\#3). $B_{x}$ continues to decrease unlil it reaches a mininum where it stans to i.rcrease again just before the exit from the electric field. 
Here $B_{x}$ is still negative when $B_{y}$ rapidly but adiabatically relums to zero (\#4). Then as the atom moves farther upward, $B_{x}$ returns to zero $(\# 5)$. It can be seen from the insen to Fig. 7 that the tip of the resultant magnetic field vector describes a closed bop in space, and this yields a contribution to $\varepsilon^{\prime}$ given by the solid angle subtended by $B$ as it traces out its path (Berry's phase) ${ }^{12}$. For $\left|B_{z}\right|>>\left|B_{x}\right|$ ivEJc|, this solid angle is just

$$
\Omega=\frac{2 \mathrm{v}\left(\mathrm{B}_{\mathrm{x}, 1}-\mathrm{B}_{\mathrm{x}, 2}\right) \mathrm{E}}{\mathrm{cB} \mathrm{B}_{\mathrm{z}}^{2}}
$$

where $B_{x, 1}$ and $B_{x, 2}$ refer to the values of $B_{x}$ at the lower and upper ends of the $E$ field region, respectively.

That this is so can be demonstrated by following the evolution of the $F=1$ wavefunction expressed in terms of "instantaneous" basis states $u_{1}, u_{0}, u_{-1}$, where the quantization axis at any given time is directed along $B$, which is a function of time $t$ in the particle rest frame. We write:

$$
\begin{gathered}
\mathrm{B}_{\mathrm{x}}=\mathrm{B} \sin \theta \cos \varphi \\
\mathrm{B}_{\mathrm{y}}=\mathrm{B} \sin \theta \sin \varphi \\
\mathrm{B}_{\mathrm{z}}=\mathrm{B} \cos \theta
\end{gathered}
$$

where $\mathrm{B}, \vartheta$, and $\phi$ depend on $\mathrm{t}$. The basis states are:

$$
u_{1}=\left(\begin{array}{c}
\frac{1}{2}(1+\cos \theta) \\
\frac{1}{\sqrt{2}} \sin \theta e^{i \varphi} \\
\frac{1}{2}(1-\cos \theta) e^{2 i \varphi}
\end{array}\right), \quad u_{0}=\left(\begin{array}{c}
-\frac{1}{\sqrt{2}} \sin \theta e^{-i \varphi} \\
\cos \theta \\
\frac{1}{\sqrt{2}} \sin \theta e^{i \varphi}
\end{array}\right), \quad u_{-1}=\left(\begin{array}{c}
\frac{1}{2}(1-\cos \theta) e^{-2 i \varphi} \\
\frac{1}{\sqrt{2}} \sin \theta e^{-i \varphi} \\
\frac{1}{2}(1+\cos \theta)
\end{array}\right)
$$

The wave function :

$$
\psi(t)=\sum_{n=-1}^{1} a_{n}(t) u_{n}(t) \exp \left(\frac{i}{\hbar} \int_{0}^{\pi} E_{n}\left(t^{\prime}\right) d t^{\prime}\right)
$$

satisfies Schroedinger's equation:

$$
H(t) \psi(t)=i \hbar \frac{\partial \psi}{\partial t}
$$

where $\mathrm{H}=-\mu \bullet B$. Inserting (11) in (12), multiplying the resulting equations on the left by $u_{m}{ }^{*}$, where $m=-1,0,1$, and dropping all rapidly oscillating terms. (adiabatic approximation), we oblain:

$$
\frac{\dot{a}_{m}}{a_{m}}=f\left(u_{m} \mid \dot{u}_{m}\right) \quad m=1,0,-1
$$

Now inserting (10) into (13) we carry out a simple calculation 10 arrive at:

$$
\varepsilon(\text { Geom })=\int_{0}^{T} \dot{\varphi}(1-\cos \theta) d t
$$


and making use of the tact that $\left|B_{2}\right| \gg>\left|B_{x}\right|,|v E / c|$, we obtain after some simple algebra:

$$
\varepsilon(\mathrm{Geom})=\frac{2 v_{x}\left(B_{x, 1}-B_{x, 2}\right) E}{c B_{2}^{2}}
$$

as expected.

Result (15) is in excellent agreement with observations. It is amusing to note that (15) contains no mention of the Bohr magneton or Planck's constant. However, it was derived in the adiabatic approximation, and if the magnetic moment and/or $\mathrm{B}_{\mathbf{z}}$ were sufficiently small or $v_{x}$ were sufficiently large this approximation would break down. Indeed we have some evidence of such a breakdown from observations of the asymmetry at extremely low $B_{\mathbf{z}^{*}}$

The quantity $\varepsilon(G e o m)$ makes a contribution to the EDM asymmetry that is E-odd and $B$-even if $B_{x, 1}-B_{x, 2}$ reverses when $B_{z}$ reverses. Indeed we have observed this in auxiliary observations, and our EDM data show evidence of a B-even, E-odd asymmetry that decreases approximately as $\mathrm{B}_{2}{ }^{-2}$. However there is the possibility of a B-odd contribution as well. if a small non-reversing inhomogeneous field $B_{x, 1}-B_{x, 2}$ exists, (possibly due to the earth's field and that of surrounding equipment) which penetrates the imnerfect shield. Note also that inspite of its dependence on $v_{x^{\prime}}$ this contribution to the EDM asymmetry would be the same for up and down beams, because $B_{x, 1}-B_{x, 2}$ must also be defined with opposite sign for up and down beams. However, in principle it can be distinguished from the true EDM asymmetry in two ways: first, by its velocity dependence, and second because it varies as $B_{z}{ }^{-2}$. In fact, making use of the latter dependence we have been able to set an upper limit to this contribution to $d_{a}$ of $2.4 \times 10^{-24} \mathrm{e} \mathrm{cm}$ for run $I$ and $1.0 \times 10^{-24} \mathrm{e} \mathrm{cm}$ for run II. (See Sec.5 for the definition of Runs 1 and li.)

\subsection{The Exv/c Elfect}

Interaction of the large magnetic moment of the thallium $6^{2} p_{1 / 2}$ state with any magnetic field that changes synchronously with the electric field can lead to a systematic asymmetry. The magnetic field includes a motional contribution from the thallium atom's passage through the intense electric field:

$$
B_{\text {mot }}=\frac{1}{c} E \times v
$$

to order v/c. Assuming that the constant electric field defines the $z$ direction, we write:

$$
|B|=\sqrt{B_{x}^{\prime 2}+B_{y}^{\prime 2}+B_{z}^{\prime 2}}
$$

where $B_{x}^{\prime}=B_{x}-E v_{y} / c, B_{y}^{\prime}=B_{y}+E v_{x} / c_{,} B_{z}^{\prime}=B_{z}$. Since $\left|v_{x}\right|>>\left|v_{y}\right|$ and $\left|B_{z}\right|>>\left|E_{x, y}\right|$, (17) may be written as: 


$$
|B|=\left|B_{z}\right|+\frac{B_{x}^{2}+B_{y}^{2}}{2 \mid B_{z}}+\frac{E}{c}\left[v_{x} \frac{B_{y}}{\left|B_{z}\right|}-v_{y} \frac{B_{z}}{B_{z}}\right]+\frac{v_{x}^{2} E^{2}}{2 c^{2} \mid B_{z}}
$$

The third term, linear in electric field, is odd in both $E$ and $B$, and could yield an EDMlike asymmetry. To a large extent this is eliminated by the use of iwo opposed beams, since they give an equal and opposite contribution if their velocities are exactly equal and opposite and if they experience the same fields. However, this ideal situation is not achieved exactly. It can be shown that at if resonance and with a full velocity distribution, the third term in (18) yields a false atomic EDM of:

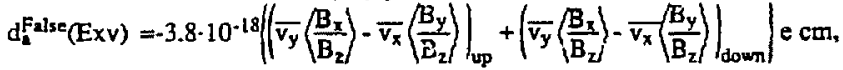

where the velocities are averaged over the velocity distribution and expressed in units of $4.3 \times 10^{4} \mathrm{~cm} / \mathrm{s}$, while

$$
\left\langle\frac{B_{x, y}}{B_{z}}\right\rangle=\frac{1}{L} \int_{0}^{L} \frac{B_{x, y}}{B_{z}} d x
$$

Let:

and

$$
\bar{v}_{x, y \text { up }}=-\bar{v}_{x, y \text { down }}+\delta \bar{v}_{x, y}
$$

$$
\left\langle\frac{B_{x, y}}{B_{z}}\right\rangle_{\text {up }}=\left\langle\frac{B_{x, y}}{B_{z}}\right\rangle_{\text {down }}+\frac{\delta B_{x, y}}{\left(B_{z}\right)} \text {, }
$$

Inserting these expressions in (19), and dropping second-order small terms and a very small $v_{y} \delta B_{x}<<B_{z}>$ contribution, we obtain:

$\mathrm{dF}^{\text {stse }}($ Exv $)=-3.8 \cdot 10^{-18}\left(\delta \bar{v}_{y}\left(\frac{B_{x}}{B_{z}}\right)-\delta \overline{v_{x}}\left(\frac{B_{y}}{B_{z}}\right)+\overline{v_{x}} \frac{\delta B_{y}}{\left(B_{z}\right)}\right) \mathrm{ecm}$.

Consider the first term of (20). In the actual experiment, we do not intentionally apply an $x$ field, but the longitudinal magnetic shielding factor is only about $2 \times 10^{3}$, and a residual component $B_{x}$ of the earth's field is non-zero in the apparatus. In addition. the $z$ and $y$ coils may be titted and thus generate an $x$ field. Furthermore, although $v_{y}$ is less than $1 \times 10^{-3} v_{x}$ we do not measure $v_{y}$. However, we can impose a known, large, $x$ field $\mathrm{B}_{\mathrm{xo}_{0}}$ and obsorve the B-odd false asymmetry. Because the first term of (20) is linear in $\mathrm{B}_{\mathbf{X}}$, the false EDM from this effect during actual runs is reduced by the ratio $B_{x} / \theta_{x o}$. We determine the residual $B_{x}$ by measuring the $r t$ resonant frequency difference between the up and down beams for $\pm B_{x_{0}}$ and for $B_{\times 0}=0$. This yields $B_{x}=2 \times 10^{-4}$ G. Thus, the possible systematic at $120 \mathrm{kHz}$ was less than $1.3 \times 10^{-24} \mathrm{e}$ $\mathrm{cm}$.

In the second term of $(20), \delta \bar{v}_{x}$ is minimized by comparing the Ramsey fringe widths of the up and down beams and adjusting the oven temperatures to equalize these widths to belter than $1 \%$. From the difference of up and down asymmetries we 
continuously measure $\angle \mathrm{B}_{\mathrm{y}} / \mathrm{B}_{\mathrm{z}}>$ and maintain this quantity at less than $1 \times 10^{-5}$ on the average. Thus the second term of $(20)$ contributes less than $3 \times 10^{-25}$ e cm to da.

The third term of $(20)$ is non zero if both beams do not experience the same $B_{y}$; this is possible if the two beams do not overlap exactly in $y$ or $z$ and the magnetic fields are inhomogeneous. Two independent techniques have been used to measure this contribution. In the first, a large magnetic field gradient at the beam axis $\left(2 B_{y} / 2 y=2.6 \times 10^{-3} \mathrm{G} / \mathrm{cm}\right)$ is deliberately imposed by means of the four inhomogensity wires mentioned earlier and the EDM asymmetry is measured. Since the actual gradient with no inhomogeneity wire current is measured to be less than $1 \times 10^{-5} \mathrm{G} / \mathrm{cm}$ (see discussion below), the result obtained for the EDM can be reduced by the corresponding ratio. The result is a contribution to $\mathrm{d}_{\mathrm{a}}$ of less than $4 \times 10^{-25} \mathrm{e} \mathrm{cm}$.

Next consider the second method. The quantity:

$$
\frac{\delta \mathrm{B}_{\mathrm{y}}}{\left\langle\mathrm{B}_{\mathrm{z}}\right\rangle}=\frac{\frac{\partial \mathrm{B}_{\mathrm{y}}}{\partial \mathrm{z}} \delta \mathrm{z}+\frac{\partial \mathrm{B}_{\mathrm{y}}}{\partial \mathrm{y}} \delta \mathrm{y}}{\left\langle\mathrm{B}_{\mathrm{z}}\right\rangle}
$$

appears in the third term of (20), where $\delta y$ and $\delta z$ are the mean relative displacements of the up and down beams in the $y$ and $z$ directions respectively. In this method we measure independently all four quantities appearing in the numerator of the right hand side of (21). Since $\nabla \times B=0$, we have $\partial \mathrm{B}_{\mathrm{y}} / \partial \mathrm{z}=\partial \mathrm{B}_{\mathrm{z}} \partial \mathrm{\partial y}$. The latter quantity is measured by employing an atomic beam that is very small in the $y$ direction, scanning it over the $y$ dimension $\Delta y=.5 \mathrm{crn}$ of the complete beam, and measuring the frequency shitt:

$$
\Delta v=\frac{\nu \partial B_{z}}{B_{z} \partial y} \Delta y
$$

This yields $\left|\partial \mathrm{B}_{\mathrm{y}} / \partial \mathrm{z}\right| \leq 3.6 \times 10^{-6} \mathrm{G} / \mathrm{cm}$. To measure $8 \mathrm{z}$ we impose a known $\partial \mathrm{B}_{\mathrm{z}} / \partial \mathrm{z}$ with the four inhomogeneity wires and determine the resulting frequency shift between up and down beams. We find that $\delta z \leq .001 \mathrm{~cm}$; this taken together with (22) implies that the contribution of the first term on the right hand side of (21) to $d_{a}$ is less than $6 \times 10^{-26} \mathrm{em}$. As for the second term in (21), the condition $\nabla \cdot B=0$ and neglect of $x$ field gradients, which are sufficiently small, permits the approximation $\partial B_{y} / \partial y=-$ $\partial \mathrm{B}_{2} / \partial z$. We then measure the resonant frequency change in either beam as we scan across its $z$ dimension with auxiliary slits. The result is $1 \partial \theta_{y} / \partial y \mid \leq 1 \times 10^{-5} \mathrm{G} / \mathrm{cm}$. To measure $\delta y$ we use two of the inhomogeneity wires to impose a large $\partial \mathrm{B}_{2} / \partial y\left(3.6 \times 10^{-4} \mathrm{G} / \mathrm{cm}\right)$ and again measure the resonant frequency difference between up and down beams. We thus obtain $\delta y \leq .001 \mathrm{~cm}$. Consequently the second term of (21) contributes less than $1.8 \times 10^{-25} \mathrm{ecm}$ to $\mathrm{d}_{\mathrm{a}}$, and the total contribution, according to the second method, is less than $2.4 \times 10^{-25} \theta \mathrm{cm}$, in agreement with the limit imposed by the first method. 
The last term in (18; can produce a small E-odd (but B-even) asymmetry if the electric field does not reverse exactly. Periodically during runs we measured the voltages on each electric field plate for each polarity and found them to be less than 3 parts in $10^{4}$. Finally, we note that the Exv effect in this experiment is complementary to the Aharonov-Casher effect observed with neutron interferometry and described by Dr. S. Werner elsetwhere in this Conterence. Both arise trom the same term in the Lagrangian.

\subsection{Other Systematics}

Charging currents can cause a false EDM in two difterent ways. First, if the peak electric field charging currents ase too large, they may generate magnetic fields that magnetize the magnetic shieiding differently for the two electric field polarities. Normally the peak charging current is $1 \mathrm{~mA}$. By taking EDM data with a peak charging current of $400 \mathrm{~mA}$, an upper limit on this contribution to $\mathrm{d}_{a}$ is obtained of $3 \times 10^{-25} \mathrm{e}$ $\mathrm{cm}$.

Charging currents may also generate an EDM-like asymmetry if the plates are not fully charged when data taking begins. This has been checked by changing the timing of the data acquisition so that a delay of only $200 \mathrm{~ms}$ is employed betore collecting data, and then data is acquired for only $400 \mathrm{~ms}$. This leads to an upper limit on this contribution to $d_{a}$ of $2 \times 10^{-25} \mathrm{ecm}$.

Electron emission trom one plate to the other or to ground will give rise to a current and a corresponding magnetic field. Since the surface structure of the two plates may differ, the electron emission and therefore the magnetic lield may depend on the electric field polarity. A test run at $125 \mathrm{kV} / \mathrm{cm}$ with leakage currents 60.200 times larger then in our normal run (where the leakage currents were $10 \mathrm{nA}$ or less) allowed us to set an upper limit on this contribution to $d_{a}$ of $8 \times 10^{-25} \mathrm{em}$.

In order to avoid a systematic due to computer control signals and groundloops we have only one ground point for the entire apparatus. We have considered other possible sources of systematic effects: phaseshift errors, different velocity distributions. different backgrounds, calibrating sensitivity error, different if power to the two loops, non-overlapping single-loop resonances, if frequency off resonance center and the acceleration (deceleration) of the down (up) beam due to gravity. None of these give a significant contribution at our present level of sensitivity. The various systematics are summarized in Table 1. 
TABLE 1.

CONTRIBUTIONS TO THE SYSTEMATIC UNCERTAINTY IN $\mathrm{d}_{\mathrm{a}}\left(10^{-24} \mathrm{ecm}\right)$

Systematic effect

Run I

Run II

Exv:

Imperfect beam overlap and magnetic field gradients

3.3

0.4

Non-zero $v_{y}$ and $B_{x}$

3.5

1.2

Velocity difference and residual $B_{y}$

0.3

0.3

Geometric Phase:

$2.4 \quad 1.0$

Leakage Currents:

0.4

0.8

Charging Currents:

High Peak

0.3

0.3

Still Flowing

0.2

0.2

Total Systematic Uncertainty*

5.4

1.9

- Calculated by combining individual contributions in quadrature

\section{RESULTS}

Two separate runs were made. The first was done at a magnetic field of .17 Gauss and $E=112 \mathrm{kV} / \mathrm{cm}$, and resulted in 51 data points, corresponding to 22 hours of integration. It yielded $d_{a}=\left(-1.1 \pm 2.5_{s t a t} \pm 5.4\right.$ syst $) \times 10^{-24}$ e $\mathrm{cm}$ with the statistical uncertainty here as elsewhere at $68 \%$ confidence intervat. Here, the systematic uncertainty was dominated by contributions due to Exv and geometric phase effects.

In the second run the uncertainties due to these effects were reduced, in the latter case by taking data at $B_{2}=.255 \mathrm{G}$. However the noise was larger and the sensitivity to a possible EDM was decreased because we employed an electric field of $100 \mathrm{kV} / \mathrm{cm}$. With 49 data points, run $I 1$ resulted in $d_{a}=\left(2.1 \pm 4.6_{s t a t} \pm 1.9_{s y s t}\right) \times 10^{-24}$ e cm, in agreement with our first measurement.

We did $a$ test run at $.085 \mathrm{G}$ in order to put a limit on the B-odd geometric phase effect and to determine the magnetic field dependence of any other contributing false effect. All the data taken at the three values of $\mathrm{B}_{2}$ show evidence of a B-even systematic proportionai to $\mathrm{B}_{2}{ }^{-2}$ :

$$
\begin{aligned}
& \left(-35.4 \pm 8.5_{\text {stat }}\right) \times 10^{-24} \mathrm{em} \text { at } .085 \mathrm{G}, \\
& \left(-7.6 \pm 2.8_{\text {stat }}\right) \times 10^{-24} \mathrm{em} \text { at } .17 \mathrm{G} \\
& \left(-3.2 \pm 4.4_{\text {stat }}\right) \times 10^{-24} \mathrm{em} \text { at } .255 \mathrm{G} .
\end{aligned}
$$


We emphasize the most important conciusion: our data give no evidence for an effect that is both B-odd and E-odd. By adding the statistical and systematic uncertainties linearly for each run, and taking the waighted average of the two runs we obtain:

$$
d_{a}=(1.6 \pm 5.0) \times 10^{-24} \mathrm{e} \mathrm{cm} \text {. }
$$

Note that except for the initial automatic rejection based on excessive noise, no rejections or cuts were made, nor were any corrections applied to the original data. Assuming an enhancement factor in the ground state of thallium of -600 , result (23) corresponds to an electron EDM of

$$
\mathrm{de}=(-2.7 \pm 8.3) \times 10^{-27} \mathrm{ecm} \text {. }
$$

The uncertainty in the electron EDM does not include the theoretical uncertainty of the enhancement factor. This result represents a significant improvement over all previous measurements ${ }^{13-17}$.

\section{ACKNOWREDGEMENTS}

We gratefully acknowiedge the excellent work of machinist $A$. Vaynberg, and also wish to thank N. Claytor, Dr. E. Garwin, Dr K. Halbach, D. Hopkins. Prof. D. McColm, N. Larkin, Prof. M. Suzuki, and A. Wydler for their many usetul contributions.

This work was supported by the U.S. Department of Energy, under contract DE-AC76SF00098, through the Lawrence Berkeley Laboratory's Experimental Research and Development Fund Program: and by the Director. OHice of Energy Research, Olfice of Basic Energy Sciences. Chemical Sciences Division; and in part by the Office of High Energy and Nuclear Physics. Division of High Energy Physics and Division of Nuclear Physics.

\section{REFERENCES}

a) Present address: Manne Siegbahn Institute, Frescativagen 24, 10405 , Stockholm, Sweden

b) Present address: Salomon Brothers, Inc., 1 New York Plaza, N.Y.,N.Y. 10004

1. S.M. Barr and A. Masiero, Phys. Rev, Lett, 58, 187, 1987

2. S.M. Barr and A. Zee, Phys. Rev. Lett. 65,21,1990

3. R.G. Leigh,S. Paban, and R.M. Xu, Univ. of Texas Theory Group Report

\#27-90,1990

4. W. Bernreuther and $M$.Suzuki, LBL Report \#27985,1990

5. LI. Schiff, Phys, Rev. 132,2194,1963

Revs. Mod. Phys, to be published

6. P.G.H. Sandars, Phys.Lett 14,194,1965

7. P.G.H. Sandars and R. M. Sternheimer, Phys. Rev. A11, 473, 1975 
8. V.V. Flambaum, Yad. Fiz (Sov J. Nuc Phys) 24, 383,1976

9. W.R.Johnson, D.S.Guo, M.ldrees, and J.Sapirstein, Phys. Rev. A 32,2093, 1985

Phys. Rev. A 34, 1043, 1986

10. E.Lindroth. A.M. Martensson-Pendrill, A.Ynnerman, and P.Oster,

J. Phys. 8 22,2447,1989

11. N.F.Ramsey, Phys. Rev. 76,996,1949: Science 248, 1612,1990

12. M.V. Berry, Proc. R. Soc. A392, 45,1984

13. S.A. Murthy, D. Krause Jr., Z.L. Li, and L.R. Hunter,

Phys. Rev. Letl. 63, 965, 1989

14. H. Gould, Phys. Rev. Lett $24,1091,1970$

15. M.C.Weisskopf, J.P.Carrico, H.Gould, E.Lipworth, and T.S. Stein,

Phys. Rev. Lelt. 21, 1645, 1968

16. S.K. Lamoreaux, J.P.Jacobs, E.R.Heckel, F.J.Raab, and E.N.Fortson,

Phys. Rey. Lett. 59, 2275.1987

17. M.A. Player and P.G.H. Sandars, J. Phys. B., 3, 1620, 1970 